\title{
Topological Properties and the Dynamical Crossover from Mixed-Valence to Kondo-Lattice Behavior in the Golden Phase of SmS
}

\author{
Chang-Jong Kang, ${ }^{1, *}$ Hong Chul Choi, ${ }^{1}$ Kyoo Kim, ${ }^{1,2}$ and B. I. Min ${ }^{1, \dagger}$ \\ ${ }^{1}$ Department of Physics, Pohang University of Science and Technology, Pohang 790-784, Korea \\ ${ }^{2} C_{-} C C M R$, Pohang University of Science and Technology, Pohang 790-784, Korea
}

(Received 9 December 2014; published 22 April 2015)

\begin{abstract}
We have investigated temperature-dependent behaviors of electronic structure and resistivity in a mixedvalent golden phase of SmS, based on the dynamical mean-field-theory band-structure calculations. Upon cooling, the coherent $\mathrm{Sm} 4 f$ bands are formed to produce the hybridization-induced pseudogap near the Fermi level, and accordingly the topology of the Fermi surface is changed to exhibit a Lifshitz-like transition. The surface states emerging in the bulk gap region are found to be not topologically protected states but just typical Rashba spin-polarized states, indicating that $\mathrm{SmS}$ is not a topological Kondo semimetal. From the analysis of anomalous resistivity behavior in SmS, we have identified universal energy scales, which characterize the Kondo-mixed-valent semimetallic systems.
\end{abstract}

DOI: 10.1103/PhysRevLett.114.166404

PACS numbers: 71.27.+a, 71.18.+y, 75.30.Mb

Kondo and mixed-valent physics in strongly correlated $4 f$-electron systems have been the subject of longstanding controversy. The subjects include various interesting phenomena such as $p$-wave superconductivity and non-Fermiliquid behavior in heavy-fermion systems [1-3], and recently proposed topological Kondo insulator behavior in a typical mixed-valent insulator $\mathrm{SmB}_{6}$ [4-14]. The topological Kondo insulator of our present interest has attracted a great deal of recent attention. However, the realization of the topological properties in $\mathrm{SmB}_{6}$ is still under debate. One immediate question is whether or not a similar mixedvalent system, SmS, also has the topological properties. Indeed, the mixed-valent golden phase of SmS $(g$-SmS) was reported to be a topological Kondo semimetal [15].

SmS has been studied for the last four decades [16-37], but there remain several issues still unresolved. SmS crystallizes in a face-centered-cubic (fcc) structure of rock-salt $(\mathrm{NaCl})$ type. At the ambient pressure, $\mathrm{SmS}$ has a so-called black phase, which is a semiconductor with indirect and direct band gaps of $90 \mathrm{meV}$ and $0.4 \mathrm{eV}$, respectively [24]. In the black phase of $\mathrm{SmS}(b-\mathrm{SmS})$, the valence state of $\mathrm{Sm}$ is divalent (2+), and so the system is nonmagnetic. Under high pressure above $6.5 \mathrm{kbar}, \mathrm{SmS}$ undergoes a first-order isostructural phase transition from $b$-SmS to $g$-SmS, in which $\mathrm{Sm}$ ions are mixed valent with the average valency of $2.6+\sim 2.8+[25,26]$. This isostructural transition is accompanied by the volume collapse by as much as 15\% [25], as shown in Fig. 1(b).

The energy gap decreases monotonically with increasing pressure. It is controversial whether $g$-SmS has a real gap or a pseudogap $[27,28]$. The resistivity behavior of $g$-SmS is quite anomalous in the sense that the overall behavior is Kondo-lattice-like but it exhibits a couple of abnormal hump structures [33-37]. Applying pressure further, $g$-SmS has a magnetic instability at about $19.5 \mathrm{kbar}$ with the antiferromagnetic order [31-33]. Above $19.5 \mathrm{kbar}$, the resistivity shows a metallic behavior and the valence state of Sm increases toward 3+ [33].

There have been several reports on the density-functionaltheory (DFT)-based band-structure study of SmS [15-17]. But even the ground-state insulating nature of $b$-SmS is not properly described by the DFT-based schemes, and so advanced methods like the dynamical mean-field theory (DMFT) should be employed to investigate the topological properties in $g$-SmS, which has strongly correlated $4 f$ electrons. Here, we have investigated electronic structures of SmS, based on the DMFT scheme. First, we have shown that the electronic properties of $b$-SmS are described properly only by the DMFT scheme. Then, we have examined the $T$-dependent electronic structure evolution in $g$-SmS. Upon cooling, the $4 f$ states form the coherent $4 f$ bands with a pseudogap feature near the Fermi level $\left(E_{F}\right)$, and accordingly the topology of the Fermi surface (FS) is changed, which is reflected well in the anomalous resistivity
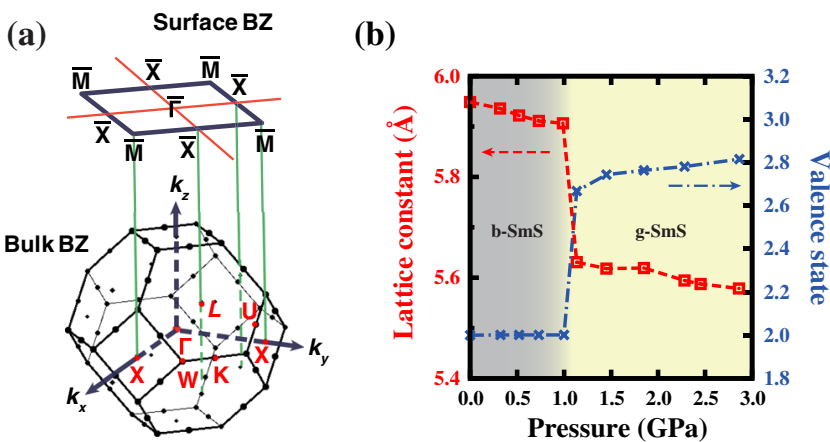

FIG. 1 (color online). Isostructural phase transition in SmS. (a) Bulk and surface Brillouin zones (BZs) of fcc SmS. (b) The pressure dependence of the lattice constant and the Sm valence state of SmS (data taken from Ref. [22]). 

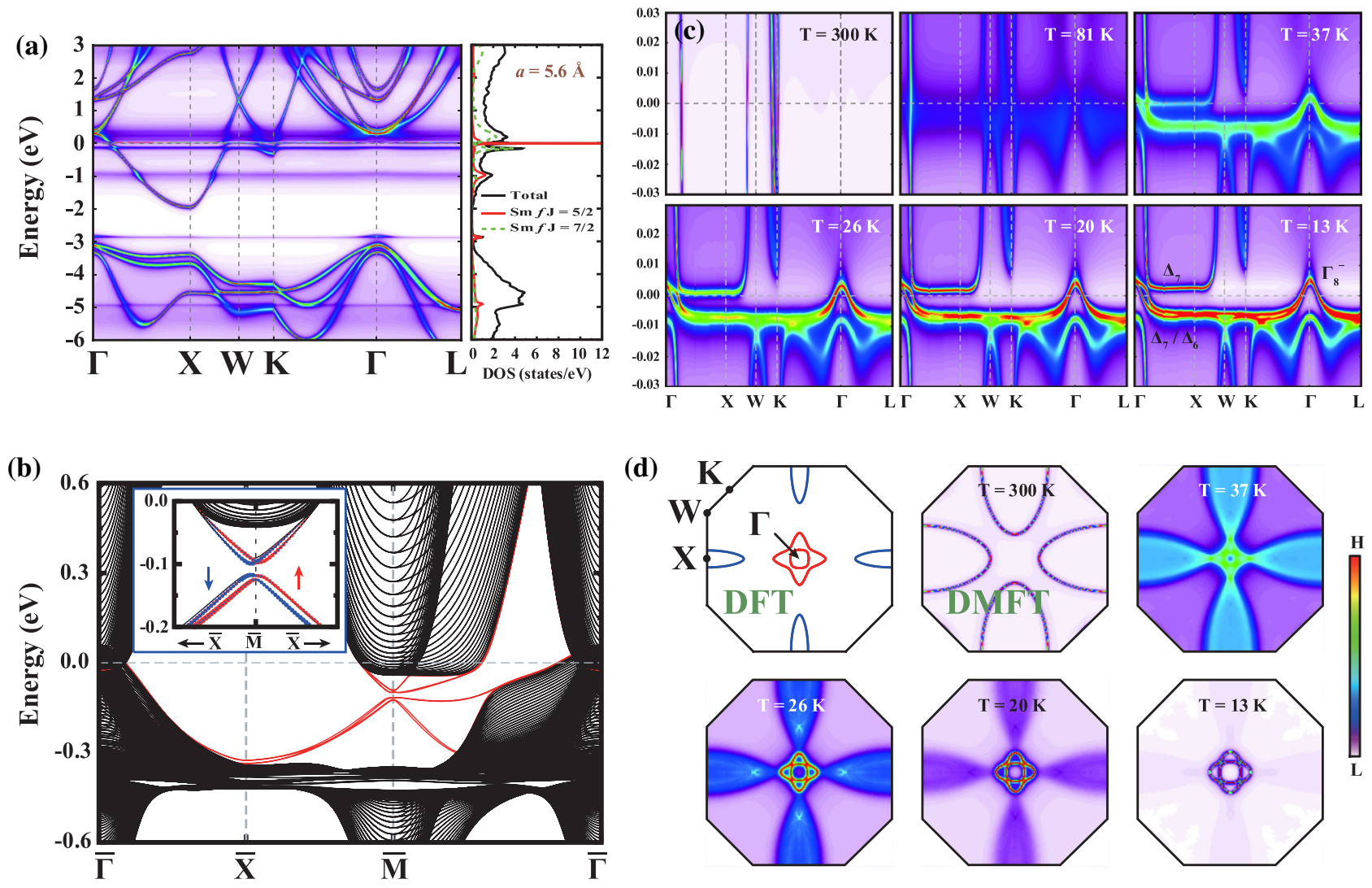

(d)
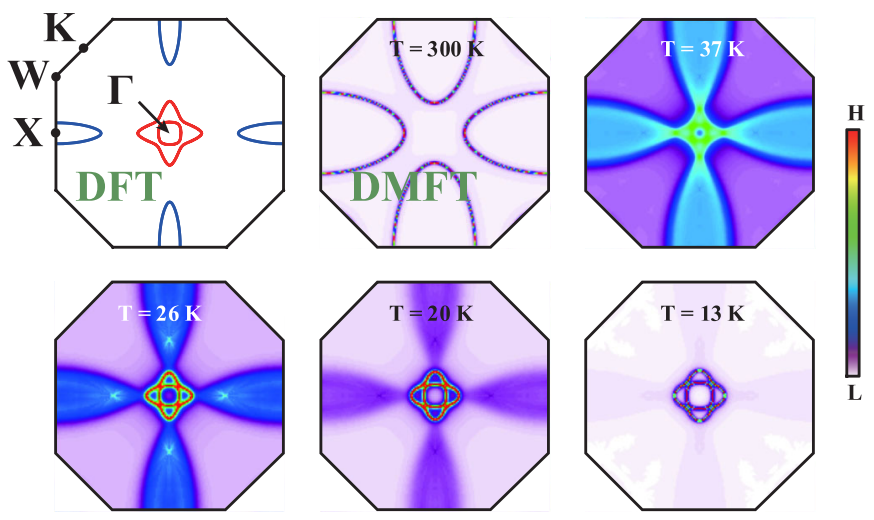

FIG. 2 (color online). Electronic structure of $g$-SmS $(a=5.6 \AA)$. (a) The DMFT band structure at $T=13 \mathrm{~K}$. (b) Surface band structure obtained by the model slab calculation based on the DFT band structure. The surface states in the inset manifest a typical Rashba splitting with a tiny gap, and so they are not topologically protected states. (c) $T$-dependent band structures in the DMFT scheme. Upon cooling, the coherent Sm $4 f$ band emerges near $E_{F}$, and the hybridization gap appears below $T=26 \mathrm{~K}$. (d) $T$-dependent FS evolution in the DMFT scheme, which is compared with the DFT FS with 10 times enhanced SOC scheme of the Sm $4 f$ electron (top left) [39]. Upon cooling, $X$-centered $d$-band pockets are reduced, while $f$-band pockets emerge at $\Gamma$.

behavior in $g$-SmS. We have demonstrated that the surface states realized in $g$-SmS are not the topological states, but are just the typical Rashba states.

We have employed the all-electron full-potential linearized augmented plane wave (FLAPW) band method implemented in the WIEN2K package [38]. We have checked that both the DFT and the DFT $+U$ (on-site Coulomb $U$ ) schemes cannot describe the ground state insulating electronic structure of SmS properly (see the Supplemental Material [39]). Therefore, we have employed the combined DFT and DMFT (DFT + DMFT) approach implemented in WIEN2K, which has successfully reproduced many aspects of the strongly correlated electron systems $[42,43]$. We used projectors in the large window of $10 \mathrm{eV}$, and the onsite Coulomb and exchange energies of $U=6.1 \mathrm{eV}, J=$ $0.8355 \mathrm{eV}$ were adopted to fit in $\mathrm{x}$-ray photoemission spectroscopy data (see Fig. S2 for the DMFT band structure of $b-\mathrm{SmS}$ in the Supplemental Material [39]) [20]. To solve the impurity problem, the symmetrized finite- $U$ noncrossing approximation (SUNCA) is used $[8,13,44,45]$. To prove the validity of the SUNCA scheme, we have also used the continuous-time quantum-Monte-Carlo scheme for
$b$-SmS and checked that the two schemes give the same result.

The DMFT band structure and DOS of $g$-SmS $(a=5.6 \AA)$ at $T=13 \mathrm{~K}$ are shown in Fig. 2(a). Notable is the flat $\operatorname{Sm} 4 f$ bands near $E_{F}$, which yield sharp Kondo resonancelike peaks in the DOS [see Fig. 3(a)]. The $4 f$ band in the vicinity of $E_{F}$ is mainly of $J=5 / 2$ character $\left(4 f_{5 / 2}\right)$, while $4 f_{7 / 2}$ bands are located at about $\pm 0.17 \mathrm{eV}$ from $E_{F}$. These coherent $\mathrm{Sm} 4 f$ bands hybridize strongly with $\mathrm{Sm} 5 d$ band to produce the hybridization gap near $E_{F}$ [see Fig. 2(c)]. However, the $4 f_{5 / 2}\left(\Gamma_{8}^{-}\right)$band at $\Gamma$ is above $E_{F}$, and so the band structure exhibits the metallic nature, having the mixed-valent state of $\mathrm{Sm}^{2.73+}$. The $\Gamma_{8}^{-}$band is dispersive with the bandwidth of about $0.03 \mathrm{eV}$, as in $\mathrm{SmB}_{6}$ [7]. The DMFT band structure near $E_{F}$ is analogous to the band structure obtained by the DFT + SOC method (SOC denotes spin-orbit coupling), as shown in the Supplemental Material [39], but the bandwidth of the former is nearly 10 times smaller than that of the latter, which is similar to the case in $\mathrm{SmB}_{6}[7,8]$.

Even though $g$-SmS has metallic nature, the band inversion occurs at $X$, and so it is tempting to anticipate 

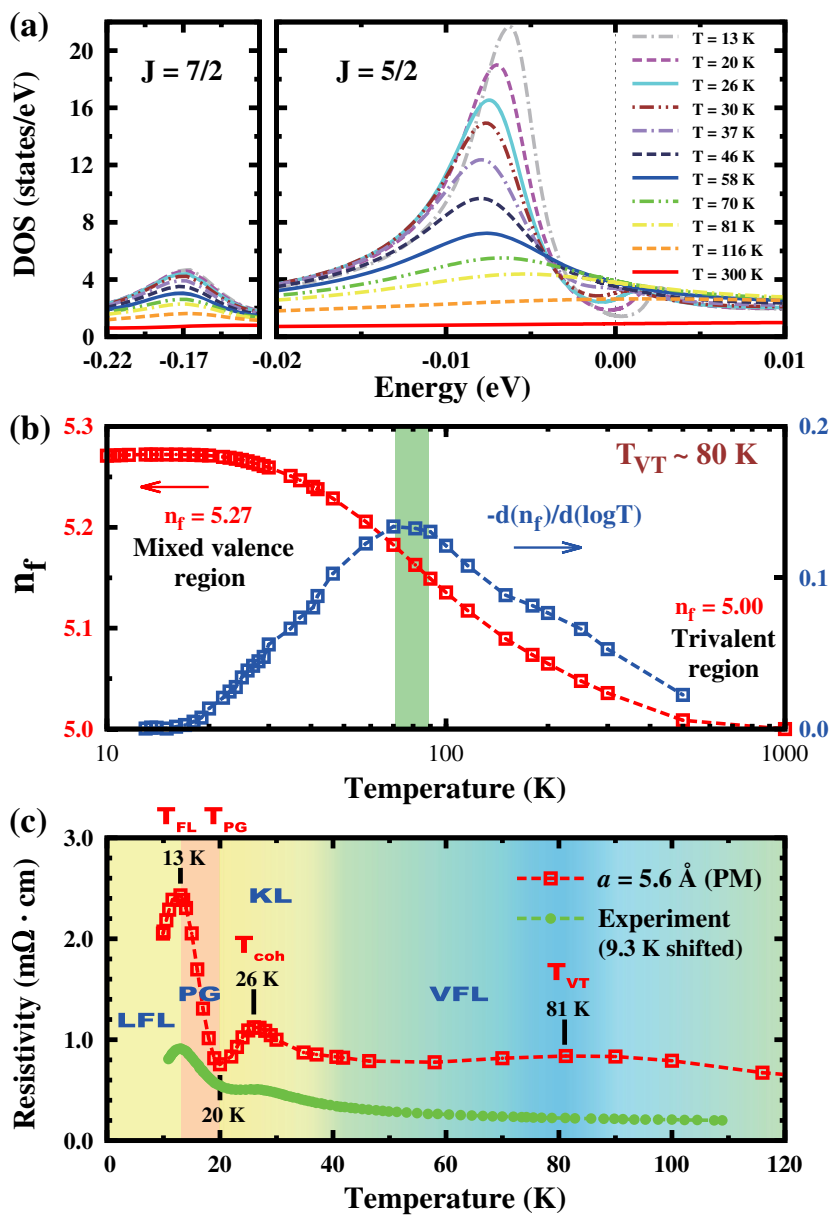

FIG. 3 (color online). $T$-dependent behaviors in $g$-SmS ( $a=5.6 \AA$ ). (a) $T$-dependent DOS variation in the DMFT scheme. Upon cooling, the $J=5 / 2$ DOS behaves like a Kondo resonance with the pseudogaplike dip feature near $E_{F}$. The $J=7 / 2$ DOS peak near $-0.17 \mathrm{eV}$ is also shown. (b) The number of occupied $f$ electrons $\left(n_{f}\right)$ as a function of the logarithmic $T$ scale. Upon cooling, $n_{f}$ increases monotonically from a trivalent state to the mixed-valent state. The effective valence transition (VT) occurs at $T \simeq 80 \mathrm{~K}$, as indicated by the peak in $-d\left(n_{f}\right) / d(\log T)$. (c) Resistivity vs $T$ obtained by the DMFT scheme. The experimental data are taken from Ref. [37], which are shifted by $9.3 \mathrm{~K}$ toward higher $T$. Upon cooling, SmS undergoes several crossover transitions from valence fluctuation liquid (VFL), Kondo liquid (KL), pseudogap (PG), to Landau Fermi liquid (LFL).

the topologically protected surface states in SmS [15]. To check the topological property, we have examined the surface band structure in Fig. 2(b), which was obtained by the model slab calculation based on the DFT bulk band structure [46]. The surface states, however, have a tiny gap instead of a Dirac cone and momentum-dependent splitting of spin states, which reveals that they are not topologically protected states but just Rashba spin-polarized surface states. This result is a contrast to that of Li et al. [15], who argued that $g-\mathrm{SmS}$ is a topological semimetal. The charge gap protection is essential to have the topological nature. In this respect, La-doped $\mathrm{SmS}$ can be a better candidate for a topological Kondo insulator, because $\mathrm{Sm}_{0.75} \mathrm{La}_{0.25} \mathrm{~S}$ under pressure was reported to be an excitonic insulator with an energy gap of $1 \mathrm{meV}$ [47].

Figure 2(c) shows the $T$-dependent band structures of $g$-SmS $(a=5.6 \AA)$. At $T=300 \mathrm{~K}$, only the $\mathrm{Sm} d$-band is seen to cut $E_{F}$. Upon cooling, Sm $4 f$ spectra start to emerge near $E_{F}$ at $T \simeq 80 \mathrm{~K}$, and $4 f_{5 / 2}$ bands are observed to be formed below $T \simeq 40 \mathrm{~K}$. At $T=26 \mathrm{~K}$, the $4 f_{5 / 2}$ hole bands near $\Gamma$ ( $\Gamma_{8}^{-}$quartet) form the coherent band, and the hybridization gap between $\operatorname{Sm} 5 d\left(t_{2 g}\right)$ and $4 f_{5 / 2}\left(\Delta_{7}\right)$ bands begins to appear at the crossing point near $\Gamma$. The coherence temperature $T_{\text {coh }}$ is usually defined in metallic Kondo lattices as an onset $T$, at which the hybridization gap appears [48]. Note that $T_{\text {coh }}$ is equivalent to $T^{*}$ that is introduced in the two-fluid model for the Kondo lattice [49]. Hence $T=26 \mathrm{~K}$ is considered to be $T_{\text {coh }}$ of $g$-SmS. In the Kondo lattice systems, $T_{\text {coh }}$ can often be identified from the peak position of the resistivity, as will be discussed in Fig. 3(c). After decreasing $T$ further, the separation between the upper $4 f_{5 / 2}$ flat band ( $\Delta_{7}$ doublet) along $\Gamma-X$ and the lower $4 f_{5 / 2}$ flat band $\left(\Delta_{7} / \Delta_{6}\right.$ quartet) becomes enhanced, and eventually the former is shifted up above $E_{F}$ at $T=20 \mathrm{~K}$. Below $T \simeq 13 \mathrm{~K}$, it is seen that the $4 f_{5 / 2}$ band becomes completely coherent over the whole Brillouin zone. In this case, the $\Gamma_{8}^{-}$hole band is still above $E_{F}$, and so $\mathrm{SmS}$ at very low $T$ would exhibit a metallic nature [see Fig. 3(c)].

Figure 2(d) presents the $T$-dependent FS evolution in $g$-SmS. Also presented is the DFT FS, for comparison, which has $X$-centered electron ellipses and $\Gamma$-centered hole pockets. At $T=300 \mathrm{~K}$, the DMFT FS arises solely from the $d$ band, which produces the $X$-centered ellipses. Upon cooling, the ellipses become reduced more and more, and so almost disappear at $T=13 \mathrm{~K}$. Their spectral weights, however, become enhanced due to the contribution from the hybridized $4 f_{5 / 2}$ band so as to have maximum intensity at $T \simeq 37 \mathrm{~K}$. On the other hand, $\Gamma$-centered hole pockets originating from the $4 f_{5 / 2}\left(\Gamma_{8}^{-}\right)$band begin to appear at $T \simeq 37 \mathrm{~K}$, and are clearly manifested below $T_{\text {coh }}=26 \mathrm{~K}$. Therefore, the topology of the FS is to be changed twice, at $T \simeq 37 \mathrm{~K}$ and $T \simeq 13 \mathrm{~K}$. The former is due to the emergence of the coherent $4 f$-band formation at $\Gamma$, while the latter is due to the separation of the upper $4 f_{5 / 2}$ flat band ( $\Delta_{7}$ doublet) from $E_{F}$.

This behavior is reminiscent of the Lifshitz transition that is a typical, continuous, quantum phase transition characterized by the topological change of the FS [50]. The feature in $\mathrm{SmS}$ is quite interesting because a Lifshitz-like transition occurs with the variation of $T$. Such a Lifshitzlike transition in $g$-SmS could be explored by ARPES and de Hass-van Alphen experiments. The change of the FS topology is well reflected in the $T$-dependent resistivity behavior in Fig. 3(c).

Upon cooling, the $4 f_{5 / 2}$ DOS peak at $\sim-6 \mathrm{meV}$ becomes sharper and sharper, as shown in Fig. 3(a), 
resembling the $T$-dependent evolution of Kondo resonance. Noteworthy is the pseudogap feature near $E_{F}$, which arises from the $f$ - $d$ band hybridization below $T \simeq 30 \mathrm{~K}$, which is close to $T_{\text {coh }}=26 \mathrm{~K}$. It is also seen that, upon cooling, the DOS at $E_{F}$ increases first and then decreases below $T \simeq 80 \mathrm{~K}$ [39]. The $4 f_{7 / 2}$ DOS near $-0.17 \mathrm{eV}$, which corresponds to the spin-orbit split sideband, behaves similarly to the $4 f_{5 / 2}$ DOS upon cooling.

The number of $f$ electrons $\left(n_{f}\right)$ in $g$-SmS vs $T$ is presented in Fig. 3(b). Upon cooling, $n_{f}$ increases monotonically from $n_{f}=5$ (trivalent state) at high $T$ to $n_{f}=$ 5.27 (mixed-valence state) at low $T$. The increasing trend of $n_{f}$ upon cooling has also been observed in $\mathrm{SmB}_{6}[13,51]$. It is worthwhile to observe that the $n_{f}$ curve has an inflection point at $T \simeq 80 \mathrm{~K}$, which indicates that the effective valence transition occurs at $T \simeq 80 \mathrm{~K}$.

Figure 3(c) shows the resistivity vs $T$ of $g$-SmS evaluated in the DMFT scheme [48]. The overall behavior is that the resistivity increases upon cooling, but decreases below $T=13 \mathrm{~K}$, as in metallic Kondo lattice systems. However, the detailed $T$-dependent behavior is quite anomalous. The calculated resistivity has a broad maximum at $T \simeq 80 \mathrm{~K}$, and starts to increase again at $T \simeq 40 \mathrm{~K}$ to produce a hump and dip structure at $T \simeq 26 \mathrm{~K}$ and $T \simeq 20 \mathrm{~K}$, respectively. Upon further cooling, the resistivity exhibits another maximum at $T=13 \mathrm{~K}$. Quite similar behavior is indeed observed in the measured resistivity of $g$-SmS [34-37], as shown in Fig. 3(c), even though the feature at $T \simeq 80 \mathrm{~K}$ is not so obvious.

The anomalous behavior of the resistivity is closely correlated with the $T$-dependent evolution of the electronic structure in Figs. 2(c) and 2(d). Namely, the broad maximum at $T \simeq 80 \mathrm{~K}$ is considered to arise from the dynamical valence fluctuation (VF), as manifested by the effective VT in Fig. 3(b) and the incoherent Sm $4 f$ spectra in Fig. 2(c) at $T \simeq 80 \mathrm{~K}$. Intriguingly, $g$-SmS has a minimum volume at $T \simeq 80 \mathrm{~K}$ [52], which signifies the close connection of a broad maximum in the resistivity with the mixed-valent nature of $\mathrm{SmS}$. Hence, we assign $T \simeq$ $80 \mathrm{~K}$ as $T_{\text {VT }}$. Interestingly, $\mathrm{SmB}_{6}$ also exhibits a similar broad maximum feature in the resistivity [53] and a minimum volume [54] near $T \simeq 150 \mathrm{~K}$. The resistivity increases below $T \simeq 40 \mathrm{~K}$, at which the coherent $4 f_{5 / 2}$ band starts to emerge in Fig. 2(c). The resistivity hump at $T \simeq 26 \mathrm{~K}$ indicates that $T_{\text {coh }}$ is around $26 \mathrm{~K}$. In conventional metallic Kondo lattices, the resistivity decreases monotonically to zero below $T_{\text {coh }}$. The behavior near $T_{\text {coh }}$ was explained in the two-fluid model by a crossover from the Kondo spin-liquid (KSL) to the Kondo Fermi liquid (KFL) [49]. We use a term of the Kondo liquid (KL) in Fig. 3(c) to comprise both KSL and KFL phases.

Thus $g$-SmS exhibits a crossover from mixed-valence to Kondo lattice behavior upon cooling [55,56]. The charge fluctuation that starts to be effective at $T \simeq 80 \mathrm{~K}$ becomes almost frozen at $T \simeq 20 \mathrm{~K}$, as shown in Fig. 3(b). Then, the spin fluctuation becomes dominating near $T \simeq 25 \mathrm{~K}$, so as to activate the Kondo screening. In fact, the effective hybridization obtained in the DMFT becomes the largest near $T=25 \mathrm{~K}$ [39].

Meanwhile, $T \simeq 20 \mathrm{~K}$ of the dip structure coincides with $T$, at which the upper $4 f_{5 / 2}$ flat band $\left(\Delta_{7}\right.$ doublet) along $\Gamma-X$ is detached from $E_{F}$. Thus the resistivity up-turn occurs due to the apparent pseudogap (PG) feature, and so we assign $T=20 \mathrm{~K}$ as $T_{\mathrm{PG}}$. Actually, this kind of humpand-dip structure in the resistivity has been observed in several Ce compounds of the Kondo insulator type, such as $\mathrm{CeNiSn}$ and $\mathrm{CeRhSb}[57,58]$. The third resistivity drop at $T=13 \mathrm{~K}$ occurs due to the complete formation of the coherent $4 f_{5 / 2}$ band over the whole Brillouin zone. Below $T=13 \mathrm{~K}$, the imaginary part of self-energy almost vanishes [39], whereby the crossover from the KL to the Landau Fermi liquid (LFL) takes place [49]. So, we assign $T=13 \mathrm{~K}$ as $T_{\mathrm{FL}}$. Therefore, $\mathrm{SmS}$ undergoes several crossover transitions upon cooling, from VF liquid (VFL), KL, PG to LFL, as shown in Fig. 3(c). The energy scales $T_{\mathrm{VT}}, T_{\mathrm{coh}}, T_{\mathrm{PG}}$, and $T_{\mathrm{FL}}$, which are identified for $\mathrm{SmS}$, are considered to be universal to characterize Kondomixed-valent semimetallic systems.

Finally, it should be pointed out that the resistivity behavior in $\mathrm{SmS}$ is quite different from that in $\mathrm{SmB}_{6} . g$ $\mathrm{SmS}$ is not a Kondo insulator but close to a Kondo semimetal, so that the conventional metallic resistivity behavior is expected to be realized at very low $T$.

In conclusion, in $g$-SmS, the coherent Sm $4 f$ bands are formed upon cooling to produce the hybridization-induced pseudogap feature near $E_{F}$, which is accompanied by a Lifshitz-like topological transition in the FS. $g$-SmS is found to be not a topological Kondo semimetal because the in-gap surface states are not topological states but are typical spin-polarized Rashba states. From the analysis of $T$-dependent resistivity of $g$-SmS, we have identified characteristic multiple energy scales, which are expected to govern the physics of Kondo-mixed-valent semimetallic systems universally.

Helpful discussions with Junwon Kim, J. H. Shim, B. H. Kim, Ki-Seok Kim, J.-S. Kang, J. D. Denlinger, J. W. Allen, and K. Sun are greatly appreciated. This work was supported by the NRF (No. 2009-0079947 and No. 2011-0025237), POSTECH BK21Plus Physics Division and Basic Science Research Institute grant, Max-Plank POSTECH/KOREA Research Initiative, and the KISTI supercomputing center (No. KSC-2013-C3-010).

*rkdc1234@postech.ac.kr bimin@postech.ac.kr

[1] G. R. Stewart, Rev. Mod. Phys. 56, 755 (1984).

[2] P. Coleman, C. Pépin, Q. Si, and R. Ramazashvili, J. Phys. Condens. Matter 13, R723 (2001).

[3] P. Gegenwart, Q. Si, and F. Steglich, Nat. Phys. 4, 186 (2008).

[4] M. Dzero, K. Sun, V. Galitski, and P. Coleman, Phys. Rev. Lett. 104, 106408 (2010). 
[5] T. Takimoto, J. Phys. Soc. Jpn. 80, 123710 (2011).

[6] M. Dzero, K. Sun, P. Coleman, and V. Galitski, Phys. Rev. B 85, 045130 (2012).

[7] C.-J. Kang, J.-W. Kim, K. Kim, J.-S. Kang, J. D. Denlinger, and B. I. Min, J. Phys. Soc. Jpn. 84, 024722 (2015).

[8] J. Kim, K. Kim, C.-J. Kang, S. Kim, H. C. Choi, J.-S. Kang, J. D. Denlinger, and B. I. Min, Phys. Rev. B 90, 075131 (2014).

[9] N. Xu et al., Phys. Rev. B 88, 121102(R) (2013).

[10] M. Neupane et al., Nat. Commun. 4, 2991 (2013).

[11] D. J. Kim, J. Xia, and Z. Fisk, Nat. Mater. 13, 466 (2014).

[12] D. J. Kim, S. Thomas, T. Grant, J. Botimer, Z. Fisk, and J. Xia, Sci. Rep. 3, 3150 (2013).

[13] J. D. Denlinger, J. W. Allen, J.-S. Kang, K. Sun, J.-W. Kim, J. H. Shim, B.I. Min, D.-J. Kim, and Z. Fisk, arXiv:1312.6637.

[14] C.-H. Min, P. Lutz, S. Fiedler, B. Y. Kang, B. K. Cho, H.-D. Kim, H. Bentmann, and F. Reinert, Phys. Rev. Lett. 112, 226402 (2014).

[15] Z. Li, J. Li, P. Blaha, and N. Kioussis, Phys. Rev. B 89, 121117(R) (2014).

[16] V. N. Antonov, B. N. Harmon, and A. N. Yaresko, Phys. Rev. B 66, 165208 (2002).

[17] A. Svane, V. Kanchana, G. Vaitheeswaran, G. Santi, W. M. Temmerman, Z. Szotek, P. Strange, and L. Petit, Phys. Rev. B 71, 045119 (2005).

[18] C. M. Varma, Rev. Mod. Phys. 48, 219 (1976).

[19] P. Villars and L. D. Calvert, Pearson's Handbook of Crystallographic Data for Intermetallic Phases (ASM International, Materials Park, 1991).

[20] M. Campagna, E. Bucher, G. K. Wertheim, and L. D. Longinotti, Phys. Rev. Lett. 33, 165 (1974).

[21] A. Jayaraman, V. Narayanamuri, E. Bucher, and R. G. Maines, Phys. Rev. Lett. 25, 1430 (1970).

[22] P. P. Deen, D. Braithwaite, N. Kernavanois, L. Paolasini, S. Raymond, A. Barla, G. Lapertot, and J. P. Sanchez, Phys. Rev. B 71, 245118 (2005).

[23] T. Ito, A. Chainani, H. Kumigashira, T. Takahashi, and N. K. Sato, Phys. Rev. B 65, 155202 (2002).

[24] T. Mizuno, T. Iizuka, S. Kimura, K. Matsubayashi, K. Imura, H. S. Suzuki, and N. K. Sato, J. Phys. Soc. Jpn. 77, 113704 (2008).

[25] M. B. Maple and D. Wohleben, Phys. Rev. Lett. 27, 511 (1971).

[26] J. M. D. Coey, S. K. Ghatak, M. Avignon, and F. Holtzberg, Phys. Rev. B 14, 3744 (1976).

[27] K. Matsubayashi, K. Imura, H. S. Suzuki, G. Chen, N. Mori, T. Nishioka, K. Deguchi, and N. K. Sato, J. Phys. Soc. Jpn. 76, 033602 (2007).

[28] P. Wachter, Handbook of the Physics and Chemistry of Rare Earths, edited by K. A. Gschneidner, L. Eyring, and S. Hüfner (North-Holland, Amsterdam, 1994), Vol. 19, p. 177.

[29] J. Flouquet, Y. Haga, P. Haen, D. Braithwaite, G. Knebel, S. Raymond, and S. Kambe, J. Magn. Magn. Mater. 272276, 27 (2004).

[30] R. M. Martin and J. W. Allen, J. Appl. Phys. 50, 7561 (1979).

[31] S. M. Shapiro, R. J. Birgeneau, and E. Bucher, Phys. Rev. Lett. 34, 470 (1975).

[32] A. Barla, J. P. Sanchez, Y. Haga, G. Lapertot, B. P. Doyle, O. Leupold, R. Rüffer, M. M. Abd-Elmeguid, R. Lengsdorf, and J. Flouquet, Phys. Rev. Lett. 92, 066401 (2004).

[33] K. Imura, K. Matsubayashi, H. S. Suzuki, N. Kabeya, K. Deguchi, and N. K. Sato, J. Phys. Soc. Jpn. 78, 104602 (2009).
[34] F. Lapierre, M. Ribault, F. Holtzberg, and J. Flouquet, Solid State Commun. 40, 347 (1981).

[35] M. Konczykowski, J. Morillo, and J. P. Senateur, Solid State Commun. 40, 517 (1981).

[36] M. Konczykowski, F. Lapierre, and P. Haen, J. Magn. Magn. Mater. 47-48, 274 (1985).

[37] K. Imura, K. Matsubayashi, H. S. Suzuki, K. Deguchi, and N. K. Sato, Physica (Amsterdam) 404B, 3028 (2009).

[38] P. Blaha, K. Schwarz, G. K. H. Madsen, D. Kavasnicka, and J. Luitz, WIEN2K (Karlheinz Schwarz, Technische Universitat Wien, Wien, Austria, 2001).

[39] See Supplemental Material at http://link.aps.org/ supplemental/10.1103/PhysRevLett.114.166404 which includes Refs. [7,18,20,23,38,40,41,44,45], for the DFT, $\mathrm{DFT}+U$, and DMFT results for $b-\mathrm{SmS}$ and $g-\mathrm{SmS}$, and $T$-dependent DMFT physical parameters.

[40] R. Pappalardo, J. Chem. Phys. 34, 1380 (1961).

[41] C. Lehner, M. Richter, and H. Eschrig, Phys. Rev. B 58, 6807 (1998).

[42] G. Kotliar, S. Y. Savrasov, K. Haule, V. S. Oudovenko, O. Parcollet, and C. A. Marianetti, Rev. Mod. Phys. 78, 865 (2006).

[43] K. Haule, C.-H. Yee, and K. Kim, Phys. Rev. B 81, 195107 (2010).

[44] K. Haule, S. Kirchner, J. Kroha, and P. Wölfle, Phys. Rev. B 64, 155111 (2001).

[45] N. Grewe, S. Schmitt, T. Jabben, and F. B. Anders, J. Phys. Condens. Matter 20, 365217 (2008).

[46] For the model slab calculation, the Wannier downfolding scheme was employed to fit the bulk band structure obtained by the DFT $+10 \times f$ SOC method (see the Supplemental Material [39]). We have also performed the DFT slab calculation and confirmed that results from the model and DFT slab calculations are essentially the same.

[47] P. Wachter, A. Jung, and P. Steiner, Phys. Rev. B 51, 5542 (R) (1995).

[48] H. C. Choi, B. I. Min, J. H. Shim, K. Haule, and G. Kotliar, Phys. Rev. Lett. 108, 016402 (2012).

[49] S. Nakatsuji, D. Pines, and Z. Fisk, Phys. Rev. Lett. 92, 016401 (2004); Y.-f. Yang and D. Pines, Phys. Rev. Lett. 100, 096404 (2008); Y.-f. Yang and D. Pines, Proc. Natl. Acad. Sci. U.S.A. 109, E3060 (2012).

[50] I. M. Lifshitz, Sov. Phys. JETP 11, 1130 (1960).

[51] M. Mizumaki, S. Tsutsui, and F. Iga, J. Phys. Conf. Ser. 176, 012034 (2009).

[52] K. Iwasa, T. Tokuyama, M. Kohgi, N. K. Sato, and N. Mori, Physica (Amsterdam) 359B-361B, 148 (2005).

[53] A. Kebede et al., Physica (Amsterdam) 223B-224B, 256 (1996).

[54] V. A. Trounov, A. L. Malyshev, D. Y. Chernyshov, M. M. Korsukova, V. N. Gurin, L. A. Aslanov, and V. V. Chernyshev, J. Phys. Condens. Matter 5, 2479 (1993).

[55] P. Coleman, in Handbook of Magnetism and Advanced Magnetic Materials, Vol. 1 Fundamentals and Theory, edited by Helmut Kronmuller and Stuart Parkin, (John Wiley and Sons, New York, 2007), pp. 95-148.

[56] P. Kumar and N. S. Vidhyadhiraja, J. Phys. Condens. Matter 23, 485601 (2011).

[57] T. Takabatake, F. Teshima, H. Fujii, S. Nishigori, T. Suzuki, T. Fujita, Y. Yamaguchi, J. Sakurai, and D. Jaccard, Phys. Rev. B 41, 9607(R) (1990).

[58] S. K. Malik and D. T. Adroja, Phys. Rev. B 43, 6277(R) (1991). 